\title{
Clinical significance of miR-138 in patients with malignant melanoma through targeting of PDK1 in the PI3K/AKT autophagy signaling pathway
}

\author{
FANJUN MENG ${ }^{1}$, YUXIA ZHANG ${ }^{2}$, XING $^{3}$, JUAN WANG ${ }^{3}$ and ZHIYU WANG ${ }^{3}$ \\ ${ }^{1}$ Department of Dermatology, Shangqiu First People's Hospital; ${ }^{2}$ Research Department, Shangqiu Medical College Shangqiu, \\ Henan 476100; ${ }^{3}$ Department of Immuno-oncology, Fourth Hospital of Hebei Medical University, \\ Shijiazhuang, Hebei 050011, P.R. China
}

Received November 22, 2016; Accepted July 10, 2017

DOI: $10.3892 /$ or.2017.5838

\begin{abstract}
The present study investigated the clinical significance of miR-138 in patients with malignant melanoma (MM), which has previously been associated with tumor growth. In patients with MM, we found that the expression of miR-138 was significantly downregulated when compared with healthy control subjects. Overexpression of miR-138 in the human melanoma cell line A2058 inhibited cell proliferation and induced cell apoptosis, and increased caspase- 3 and Bax protein expression when compared with a negative control group. Meanwhile, miR-138 overexpression promoted cell autophagy, induced LC3 protein expression, and suppressed the PI3K/AKT/mTOR signaling pathway and PDK1 protein expression in A2058 cells. LY294002, an inhibitor of PI3K, suppressed PI3K/AKT/mTOR signaling, induced apoptosis, inhibited cell proliferation, increased caspase- 3 and Bax protein expression, and decreased PDK1 protein expression in A2058 cells following miR-138 overexpression. Collectively, our findings indicate the clinical significance of miR-138 in patients with MM through its targeting of PDK1 expression in the PI3K/AKT/mTOR autophagy signaling pathway.
\end{abstract}

\section{Introduction}

Malignant melanoma (MM) is a type of highly malignant tumor that primarily develops in adults over the age of 30 years, and is associated with a high rate of mortality (1). The morbidity rate of MM, which has gradually increased over recent years, particularly in younger generations, accounts for $\sim 2 \%$ of all malignant tumors, and ranks third among skin malignancies

Correspondence to: Professor Zhiyu Wang, Department of Immuno-oncology, Fourth Hospital of Hebei Medical University, 12 Jiankang Road, Shijiazhuang, Hebei 050011, P.R. China E-mail: wrfgo394555@126.com

Key words: miR-138, malignant melanoma, autophagy, PI3K/AKT, PDK1
(6-20\% of skin malignancies) (2). MM can occur on any region of the skin, with skin regions exposed to the highest levels of friction at the extremities being the most vulnerable, and is characterized by early metastasis, aggressive invasion and poor prognosis (3). Current treatment strategies for MM typically involve surgery followed by biotherapy, radiotherapy and/or chemotherapy. However, these methods have to date yielded unsatisfactory clinical effects on the prognosis and mortality rate of patients with MM (4).

Due to the development of molecular biology techniques over recent years, research into the genetics and molecular biology of MM, including its genesis, development and metastasis, has made great progress, which may aid in improving clinical treatment. However, there has been little research into the effects and underlying mechanisms of microRNAs (miRNAs/miRs) in MM $(5,6)$.

miRNAs are types of small non-coding RNAs of 18-25 nucleotides in length that have recently been associated with MM (6). miRNAs serve important regulatory roles in the normal physiological functioning of the body by regulation of target gene expression, which can influence protein expression. Notably, under certain conditions, increased or decreased expression of specific target proteins can result in cell functional changes and tumorigenesis (7). The expression profiles of miRNAs greatly differ between tumor cells and normal tissue cells (8). Increased, decreased or depleted expression levels of certain miRNAs can be observed in the majority of tumor cells of various types, which is an important mechanism underlying changes in the proliferation and growth of tumor cells (9). Therefore, in the present study miR-138 was selected as an miRNA detected in MM, and its putative target genes and function were predicted. Preliminary investigations into the molecular mechanisms of miR-138 in the proliferation and growth of MM were also conducted.

Autophagy (from the Greek for 'self-eating') is a cell phenomenon in which the intracellular lysosome engulfs and degrades damaged or redundant organelles and mutant proteins (10). The autophagy-related genes are critical factors that regulate the process of autophagy (10). The primary functions of autophagy are to eliminate biological waste materials within the body and to promote metabolism by 
degradation of damaged organelles under stress conditions, such as hunger, which aids in the self-protection of cells and promotes cell survival (11). However, excessive autophagy decreases cell survival by promoting autophagic cell death (11).

Numerous signal transduction pathways are associated with autophagy, including PI3K/AKT/mTOR, Ras/PKA and Jnk (12). Each pathway promotes or inhibits the process of autophagy through various mechanisms (13). At present, the autophagy-associated signaling pathway PI3K/AKT/mTOR has been the most widely studied (13). Cell autophagy is regulated by mTOR, and is mainly activated by inhibition of the PTEN protein. The PI3K/AKT/mTOR signal transduction pathway can act against the process of cell autophagy, which disrupts cellular homeostasis and proliferation; however, $\mathrm{PI} 3 \mathrm{~K} / \mathrm{AKT} / \mathrm{mTOR}$ signaling may also increase the survival of tumorigenic cells by preventing autophagic programmed cell death, which facilitates tumor metastasis (14).

Phosphoinositide-dependent kinase 1 (PDK1) is a type of serine/threonine protein kinase with a molecular weight of $63 \mathrm{kDa}$, and contains a PH domain that binds with the inositol triphosphate (PIP3) product of PI3K, which enables PDK1 to target the cell membrane and activate AKT. Meanwhile, other substrates of PDK1, with an inability to target the cell membrane or lack of a PH domain, can also directly bind with PDK1 to become activated (15). PDK1 itself can be activated by bivalent polymerization and cross phosphorylation (16). PDK1 serves as an upstream kinase of AKT, and catalyzes the phosphorylation of Thr308 within the activation loop of AKT, stimulating a $>30$-fold increase in AKT activity. Such activation is PIP3- or PIP2-dependent, thus giving rise to the name PDK1 (17). As a common link between multiple important signaling pathways in cells, PDK1 is widely expressed in many human tissues, and plays a critical role in a number of tumor processes, including tumor cell growth, protection against cell apoptosis, stimulation of the epithelial-mesenchymal transition (EMT) and tumor angiogenesis (17). The present study, aimed to elucidate the clinical significance of miR-138 in patients with MM, as well as its underlying mechanisms in MM cells.

\section{Materials and methods}

Patients and blood samples. The present study was approved by the Ethics Committee of Shangqiu First People's Hospital (Shangqiu, China), and written informed consent was obtained from all the patients. Whole blood samples from 5 patients with MM and 6 healthy volunteers were collected from the Department of Dermatology, Shangqiu First People's Hospital. Serum was isolated after centrifugation at 2,000 $\mathrm{x}$ g for $10 \mathrm{~min}$ at room temperature. A follow-up of all patients was conducted every 3 months by telephone or reexamination at the clinic, and the overall survival (OS) and disease-free survival (DFS) rates of the MM patients with high and low miR-138 expression were subsequently determined.

Cell lines and culture. The human MM cell line A2058 was purchased from the Cell Bank of Central South University (Shanghai, China) and cultured in Dulbecco's modified Eagle's medium (DMEM) with $10 \%$ fetal bovine serum (FBS; Thermo Fisher Scientific, Inc., Waltham, MA, USA) at $37^{\circ} \mathrm{C}$ and $5 \% \mathrm{CO}_{2}$.
$R N A$ isolation and $R T-q P C R$. Total RNA was extracted from serum and cells using TRIzol reagent (Thermo Fisher Scientific, Inc.). cDNA was synthesized from total RNA using a MiRNA Reverse Transcription kit (Qiagen, Inc., Valencia, CA, USA) under the following conditions: $16^{\circ} \mathrm{C}$ for $30 \mathrm{~min}$, followed by $42^{\circ} \mathrm{C}$ for $30 \mathrm{~min}$ and enzyme inactivation at $85^{\circ} \mathrm{C}$ for $5 \mathrm{~min}$. The expression of miR-138 was subsequently assessed using a 7500 Fast Real Time PCR System (Thermo Fisher Scientific, Inc.). The PCR cycling conditions were $95^{\circ} \mathrm{C}$ for $5 \mathrm{~min}$, followed by 40 cycles of denaturation at $95^{\circ} \mathrm{C}$ for $30 \mathrm{sec}$ and $60^{\circ} \mathrm{C}$ for $45 \mathrm{sec}$. The relative expression of miRNA was determined using the $2^{-\Delta \Delta C q}$ method.

Transfection. miR-138 mimics and negative control miRNAs were synthesized by Sangon Biotech Co., Ltd. (Shanghai, China). A2058 cells were transfected with $100 \mathrm{ng}$ of miR-138 mimics or negative control miRNA using Lipofectamine 2000 (Thermo Fisher Scientific, Inc.), in accordance with the manufacturer's instructions. At $6 \mathrm{~h}$ after transfection, a subgroup of cells was treated with $100 \mathrm{nM}$ of LY294002 (Selleck Chemicals, Shanghai, China) for $48 \mathrm{~h}$ to inhibit PI3K.

Cell proliferation and apoptosis assays. At $48 \mathrm{~h}$ after transfection, cells were plated into a 96 -well plate $(10,000$ cells/well), and $20 \mu \mathrm{l}$ of MTT reagent $(5 \mathrm{mg} / \mathrm{ml}$; Sigma-Aldrich, St. Louis, MO, USA) was added to each well and incubated for $4 \mathrm{~h}$. The supernatant was removed and $100 \mu$ l dimethyl sulfoxide DMSO (Beyotime Institute of Biotechnology, Haimen, China) was added and incubated for $20 \mathrm{~min}$. The absorbance was detected using an ELx800 absorbance microplate reader (BioTek Instruments, Inc., Winooski, VT, USA) at $492 \mathrm{~nm}$.

In addition, at $48 \mathrm{~h}$ after transfection, the cells were plated into a 6 -well plate $\left(1 \times 10^{6}\right.$ cells/well) and washed 3 times with phosphate-buffered saline (PBS). Cells were then stained using an Annexin V-FITC/PI kit (Nanjing KeyGen Biotech Co., Ltd., Nanjing, China). The rates of apoptosis were assessed using a flow cytometer (Bio-Rad Laboratories, Inc., Hercules, CA, USA).

Western blot analysis and caspase-3 activity assay. At $48 \mathrm{~h}$ after transfection, cells were lysed in cold RIPA buffer and protein concentration was determined using a BCA protein assay kit (both from Beyotime Institute of Biotechnology). A total of 30-60 $\mu \mathrm{g}$ of protein was then separated using 8-10\% SDS-PAGE and transferred to a polyvinylidene fluoride (PVDF) membrane (Thermo Fisher Scientific, Inc.). The PVDF membrane was blocked in 5\% non-fat dried milk in TBST (Thermo Fisher Scientific, Inc.) for 1-2 $h$, and then incubated with primary antibodies against LC3, Bax, p62, PI3K, p-AKT, p-mTOR, p-PDK1 and GAPDH overnight at $4^{\circ} \mathrm{C}$. After 3 washes with PBS, the PVDF membrane was incubated with an anti-rabbit secondary antibody for $1 \mathrm{~h}$ at room temperature. Immunolabelled bands were detected using an ECL Western Blotting kit (Beyotime Institute of Biotechnology) and analyzed using Image-Pro Plus software, version 6.0 (Media Cybernetics, Inc., Rockville, MD, USA).

In addition, a total of $5 \mu \mathrm{g}$ of protein was used to assess caspase- 3 activity using a caspase- 3 activity kit (Beyotime Institute of Biotechnology), and the absorbance was assessed using an ELx800 absorbance microplate reader at $405 \mathrm{~nm}$. 


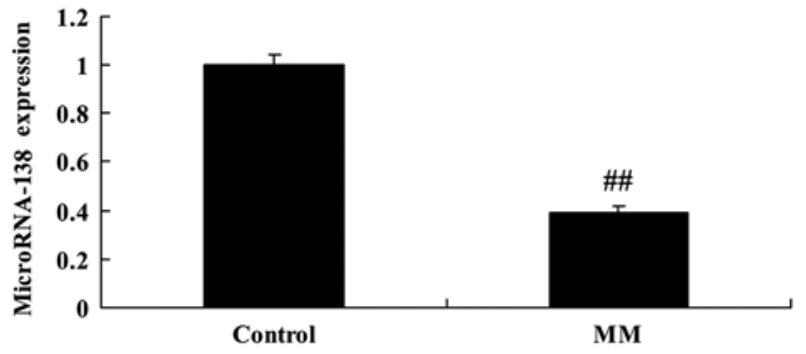

Figure 1. miR-138 expression in patients with MM. ${ }^{\# \#} \mathrm{p}<0.01$ vs. the control group. Control, control group; MM, malignant melanoma.

Fluorescence microscopy. At 48 h after transfection, A2058 cells $\left(1 \times 10^{3}\right.$ cells $\left./ \mathrm{ml}\right)$ were seeded onto cell chamber slides and washed 3 times with PBS. The cells were then fixed with $4 \%$ formaldehyde at $4^{\circ} \mathrm{C}$ for $30 \mathrm{~min}$ and stained with anti-LC3 (1:200; Santa Cruz Biotechnology, Inc., Dallas, TX, USA) at $4^{\circ} \mathrm{C}$ overnight. The cells were then incubated with an anti-rabbit secondary antibody (1:1,000; Bio-Rad Laboratories, Inc.) at room temperature for $3 \mathrm{~h}$, and subsequently stained with DAPI at room temperature for $30 \mathrm{~min}$. The expression of the LC3 protein was visualized by fluorescence microscopy using an AxioScope A1 Microscope (Zeiss GmbH, Jena, Germany).

Statistical analysis. All data are expressed as the mean \pm SD. Statistical analysis of differences was performed using one-way analysis of variance (ANOVA) and $\mathrm{P}<0.05$ was considered to indicate a statistically significant difference.

\section{Results}

miR-138 expression in MM patients and cells. Firstly, we analyzed the expression of miR-138 in patients with MM using RT-qPCR. In patients with MM, we found that miR-138 expression was significantly downregulated when compared with healthy control subjects (Fig. 1).

$O S$ and DFS rates are associated with miR-138 expression. Next, the OS and DFS rates of MM patients with high and low miR-138 expression were determined. The OS and DFS rates of $\mathrm{MM}$ patients with high miR-138 expression were markedly lower than those of patients with low miR-138 expression (Fig. 2).

miR-138 overexpression inhibits proliferation and induces apoptosis in A2058 cells. We investigated the effects of miR-138 on the growth and apoptosis of MM cells. As shown in Fig. 3A, miR-138 mimics were transfected into A2058 cells, which increased the expression of miR-138 in A2058 cells when compared with cells transfected with negative control mimics. Overexpression of miR-138 significantly inhibited proliferation, induced apoptosis and increased caspase- 3 activity in A2058 cells when compared with the negative control group (Fig. 3B-D).

miR-138 overexpression induces LC3 and Bax protein expression in A2058 cells. Subsequently, western blot analysis was performed to evaluate the protein expression of LC3 and Bax. As shown in Fig. 4, overexpression of miR-138 significantly induced LC3 and Bax protein expression in A2058 cells compared with the negative control group.

miR-138 overexpression suppresses PI3K/PDK1/AKT/mTOR signaling in A2058 cells. To investigate the relationship between miR-138 and the PI3K/PDK1/AKT/mTOR signaling pathway in MM, the effect of miR-138 on the activation of $\mathrm{PI} 3 \mathrm{~K} / \mathrm{AKT} / \mathrm{mTOR}$ signaling in A2058 cells was investigated by western blot analysis. Fig. 5 shows that miR-138 overexpression significantly suppressed PI3K/AKT/mTOR signaling in A2058 cells when compared with the negative control group.

PI3K suppression enhances the effects of miR-138 overexpression on PI3K/PDK1/AKT/mTOR signaling. The functions of miR-138 and the PI3K/PDK1/AKT/mTOR signaling pathway in the regulation of A2058 cells were investigated. As shown in Fig. 6, the PI3K inhibitor LY294002 enhanced the inhibitory effects of miR-138 overexpression on PI3K/AKT/mTOR signaling in A2058 cells, relative to cells with only miR-138 overexpression.

PI3K suppression enhances the effects of miR-138 overexpression on the proliferation and apoptosis of A2058 cells. We then evaluated in vitro whether PI3K was involved in the effects
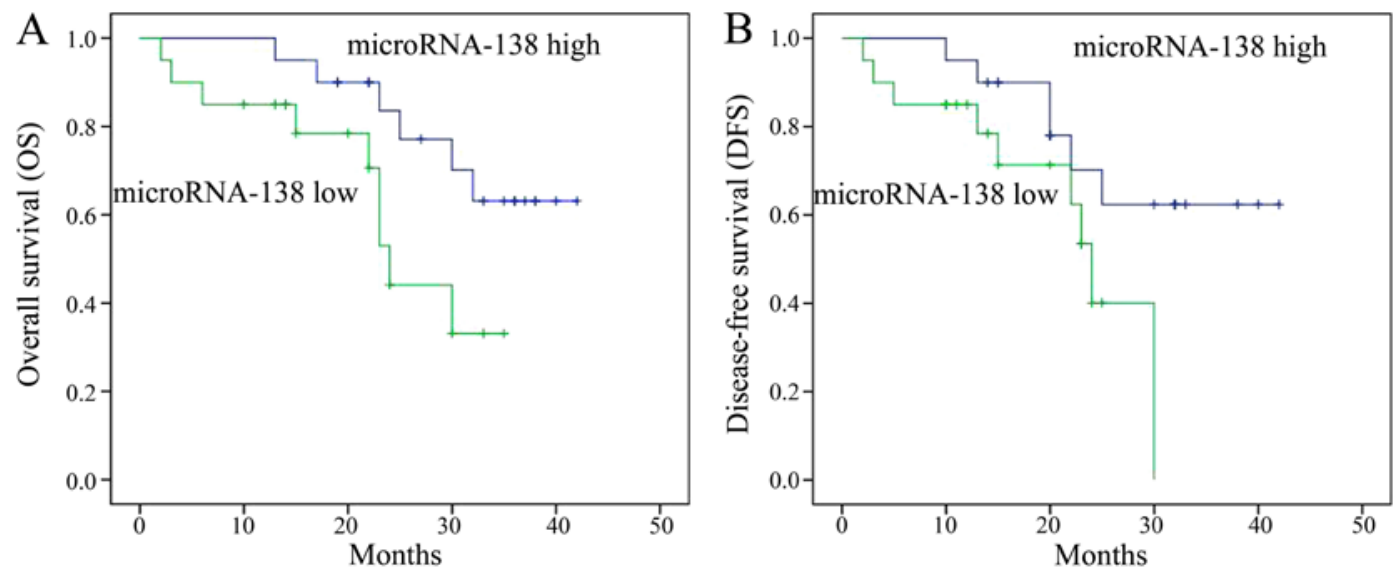

Figure 2. Overall and disease-free survival of MM patients with high and low miR-138 expression. MM, malignant melanoma. 

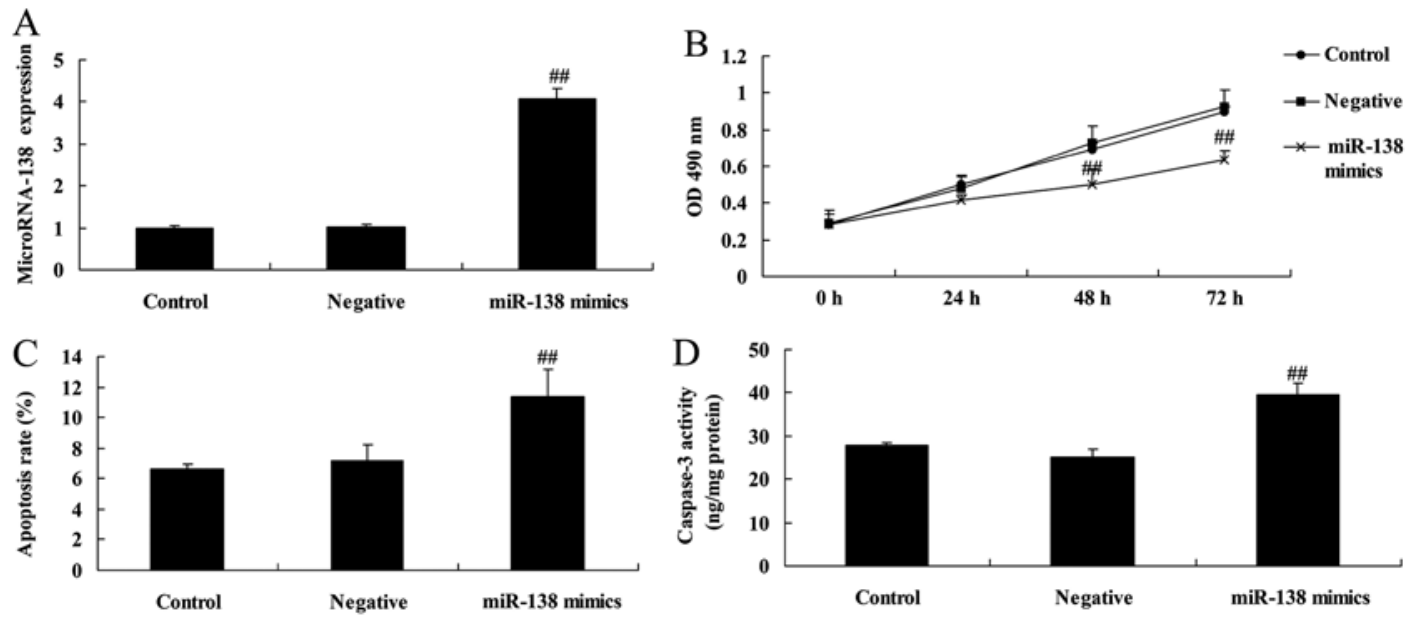

Figure 3. miR-138 overexpression inhibits proliferation and induces apoptosis in A2058 cells. (A) miR-138 overexpression inhibited (B) cell proliferation, and induced (C) apoptosis, and (D) caspase-3 activity in A2058 cells; ${ }^{\# \#}$ p $<0.01$ vs. the control group. Control, control group; negative, negative control group; miR-138 mimics, miR-138 overexpression group.
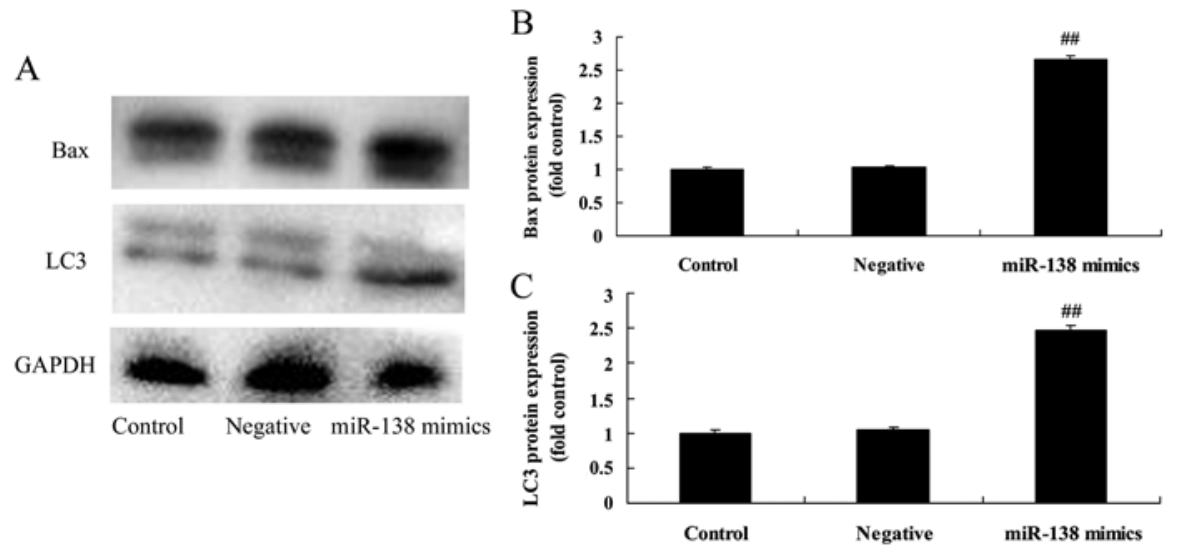

Figure 4. miR-138 overexpression induces LC3 and Bax protein expression in A2058 cells. (A) Results of western blot analysis indicated that miR-138 overexpression induced LC 3 and Bax protein expression in A2058 cells. Statistical analysis of (B) LC 3 and (C) Bax protein expression; ${ }^{\# \#}$ p $<0.01$ vs. the control group. Control, control group; negative, negative control group; miR-138 mimics, miR-138 overexpression group.
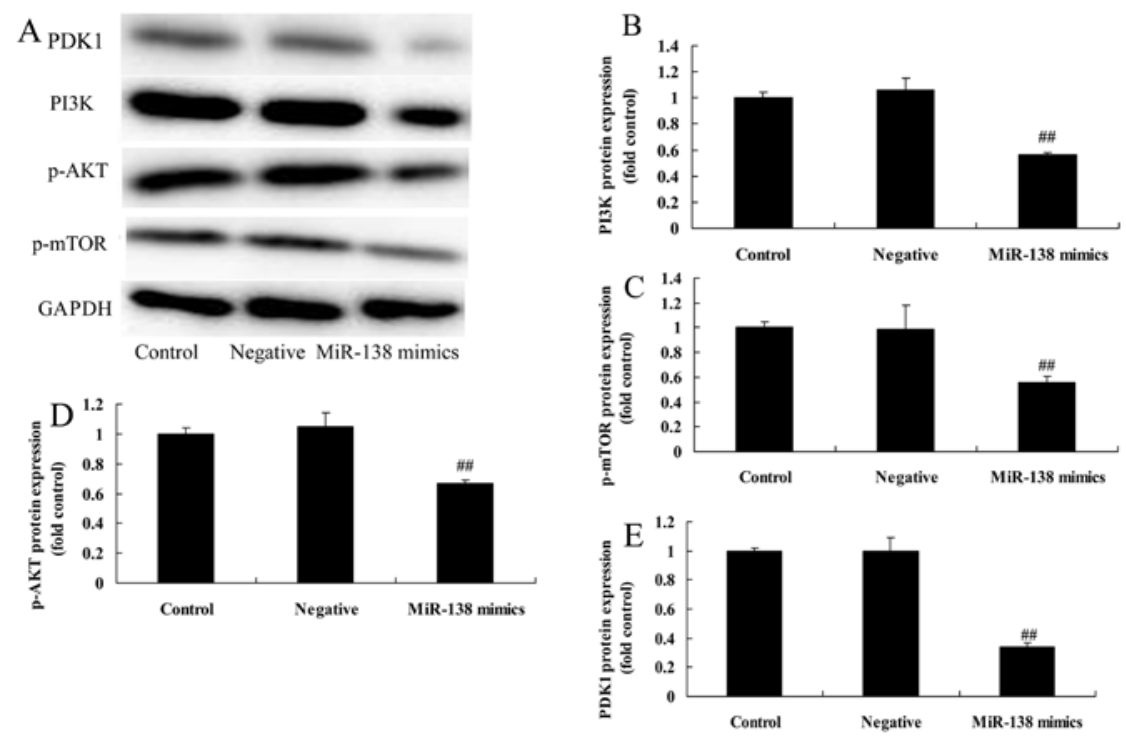

Figure 5. miR-138 overexpression suppresses the PI3K/PDK1/AKT/mTOR signaling pathway in A2058 cells. (A) Results of western blot analysis indicated that miR-138 overexpression suppressed PI3K, p-AKT and p-mTOR protein expression in A2058 cells. Statistical analysis of (B) PI3K, (C) p-AKT and (D) p-mTOR and (E) PDK1 expression; \# $\mathrm{p}<0.01$ vs. the control group. Control, control group; negative, negative control group; miR-138 mimics, miR-138 overexpression group. 

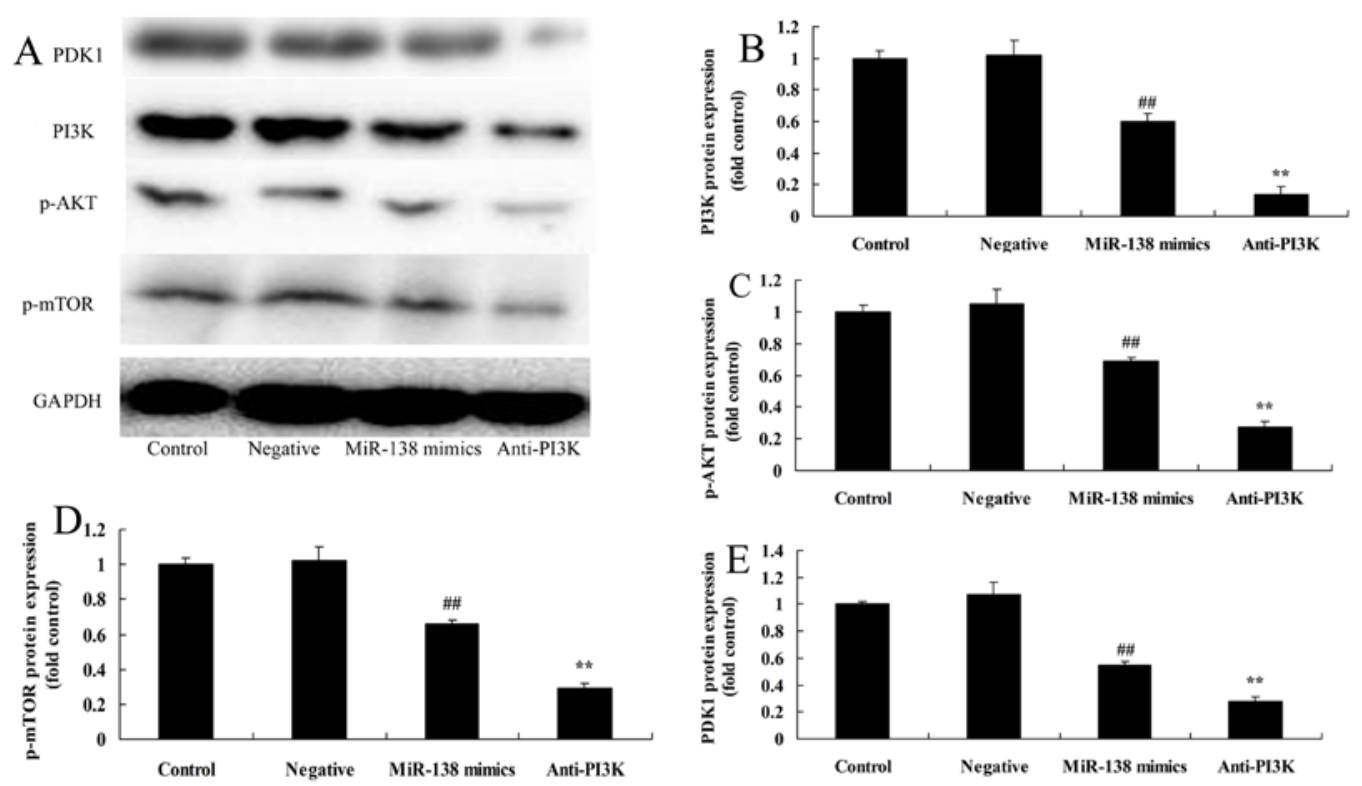

Figure 6. PI3K suppression further suppresses PI3K/PDK1/AKT/mTOR signaling in A2058 cells following miR-138 overexpression. (A) Western blot analysis indicated that PI3K suppression further inhibited PI3K, p-AKT and p-mTOR protein expression in A2058 cells following miR-138 overexpression. Statistical analysis of (B) PI3K, (C) p-AKT and (D) p-mTOR and (E) PDK1 expression; ${ }^{\# \#}$ p $<0.01$ vs. the control group, ${ }^{* *}$ p $<0.01$ vs. the control group. Control, control group; negative, negative control group; miR-138 mimics, miR-138 overexpression group; anti-PI3K, PI3K inhibitor (LY294002) and miR-138 overexpression group.

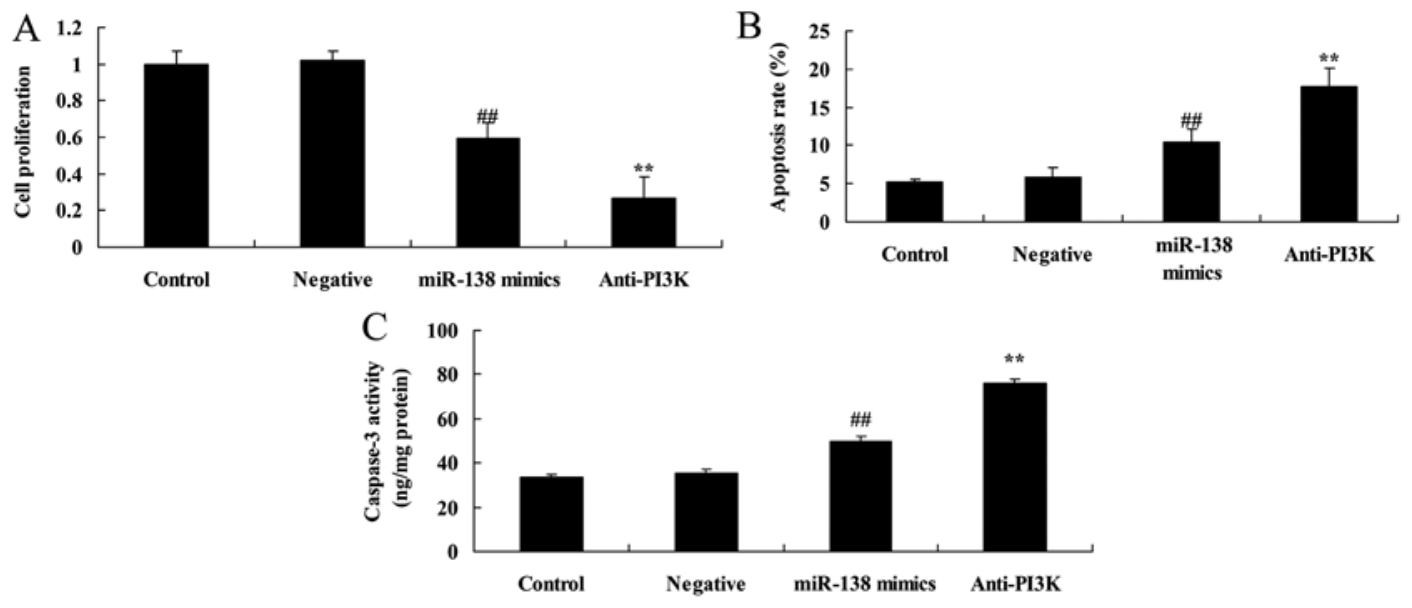

Figure 7. PI3K suppression influences proliferation and apoptosis in A2058 cells following miR-138 overexpression. PI3K suppression further decreased (A) cell proliferation and induced (B) apoptosis, and (C) caspase-3 activity in A2058 cells following miR-138 overexpression; ${ }^{* \#}$ p $<0.01$ vs. the control group, ${ }^{* *} \mathrm{p}<0.01$ vs. the control group. Control, control group; negative, negative control group; miR-138 mimics, miR-138 overexpression group; anti-PI3K, PI3K inhibitor (LY294002) and miR-138 overexpression group.

of miR-138 on the proliferation and apoptosis of A2058 cells. The data in Fig. 7 indicated that the PI3K inhibitor significantly decreased cell proliferation while promoting apoptosis and caspase-3 activity in A2058 cells following miR-138 overexpression, relative to the miR-138 overexpression-only cell group.

PI3K suppression enhances the effects of miR-138 overexpression on LC3 and Bax protein expression in A2058 cells. Furthermore, we determined whether PI3K was involved in the effects of miR-138 on the protein expression of LC3 and Bax in A2058 cells. We used laser confocal scanning microscopy to analyze LC3 expression, and observed that PI3K suppression significantly promoted LC3 protein expression in A2058 cells following miR-138 overexpression, relative to the miR-138 overexpression-only cell group (Fig. 8). Similarly, western blot analysis indicated that PI3K suppression significantly increased LC3 and Bax protein expression in A2058 cells following miR-138 overexpression, relative to the miR-138 overexpression-only cell group (Fig. 9).

\section{Discussion}

MM is a type of highly malignant tumor that predominantly occurs in adults over the age of 30 years, and is associated with a high rate of mortality (18). The morbidity rate of MM, which has gradually increased over recent years, particularly in younger generations, accounts for $\sim 2 \%$ of all malignant 

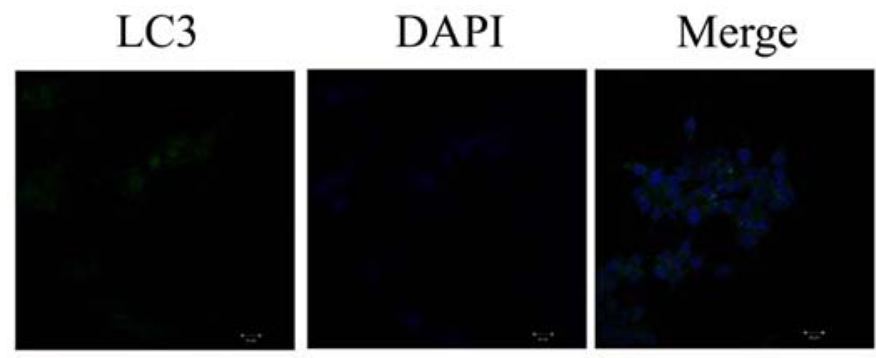

\section{Control}
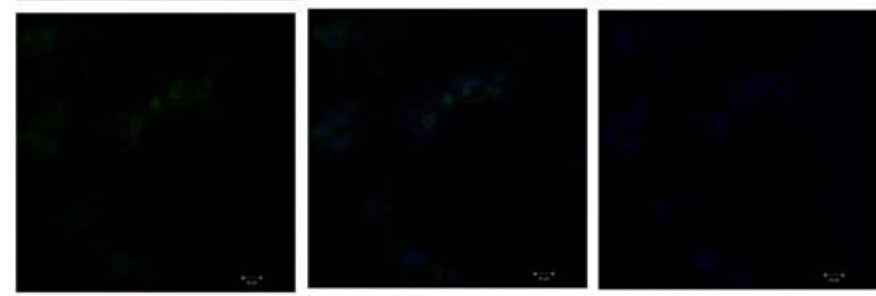

\section{Negative}
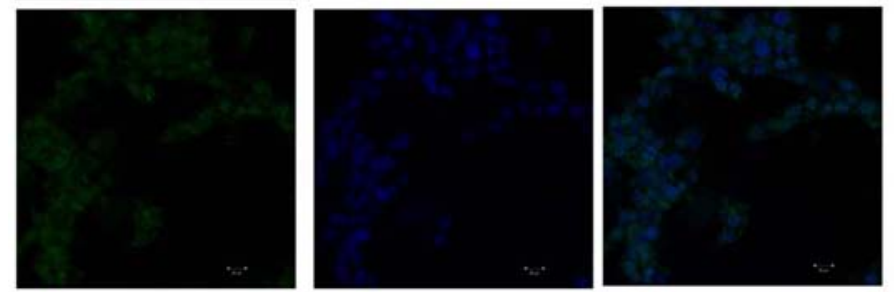

\section{$\operatorname{miR}-138$}

mimics
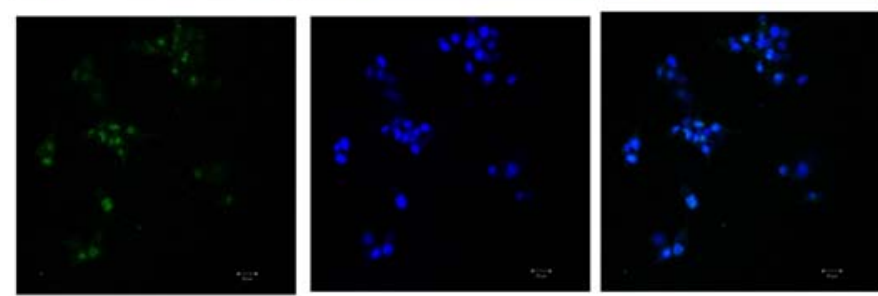

\section{Anti-PI3K}

Figure 8. Laser confocal scanning microscopy of LC3 expression.

A

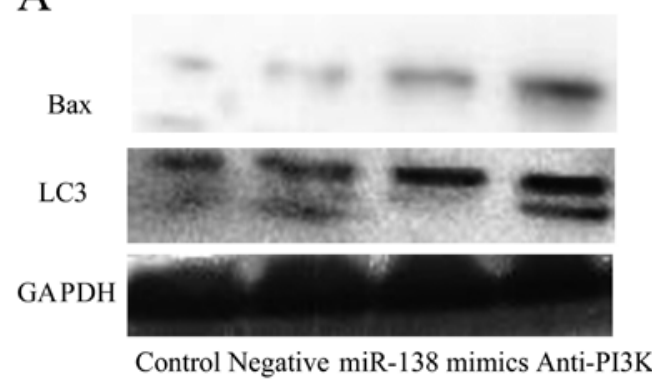

Figure 9. PI3K suppression influences LC3 and Bax protein expression in A2058 cells following miR-138 overexpression. (A) Western blot analysis showed that PI3K suppression further increased LC3 and Bax protein expression in A2058 cells following miR-138 overexpression. Statistical analysis of (B) LC3 and (C) Bax protein expression; ${ }^{\# /} \mathrm{p}<0.01$ vs. the control group, ${ }^{* * *} \mathrm{p}<0.01$ vs. the control group. Control, control group; negative, negative control group; miR-138 mimics, miR-138 overexpression group; anti-PI3K, PI3K inhibitor (LY294002) and miR-138 overexpression group.

tumors, and ranks third among skin malignancies $(6-20 \%$ of skin malignancies) (19). MM can occur on any region of the skin, with skin regions exposed to friction at the extremities being most vulnerable, and is characterized by early metastasis, aggressive invasion and poor prognosis (20). Current treatment strategies for MM typically involve surgery followed by 
biotherapy, radiotherapy and/or chemotherapy, although these methods have to date yielded unsatisfactory clinical effects on the prognosis and mortality rate of patients (21). In the present study the results revealed that miR-138 expression was significantly downregulated in patients with $\mathrm{MM}$ and the MM cell line A2058 when compared with normal controls. Furthermore, the OS and DFS rates of MM patients with high miR-138 expression were observably lower than those of patients with low miR-138 expression.

Cell autophagy can not only eliminate the metabolic products of cells and abnormal intracellular proteins, but can also degrade redundant or damaged organelles (11). Autophagy can be induced during early newborn development, hypoxia, infection and hunger, and its degradation products can provide materials and energy for cells, thus maintaining cell homeostasis (22). However, mutant, damaged or aging cells can undergo programmed cell death as a result of autophagy (10). The present results revealed that miR-138 overexpression significantly inhibited cell proliferation, induced apoptosis, increased caspase- 3 activity and induced LC3 and Bax protein expression in A2058 cells. Stojcheva et al (23) revealed that miR-138 induced acquired alkylator resistance by the induction of autophagy.

The PI3K/AKT signal transduction pathway is principally regulated by the tumor suppressor genes PTEN and SHIP2. PTEN and SHIP2 dephosphorylate PI(3,4,5)-P3 at the $3^{\prime}$ and 5' positions, respectively, and as a result, $\mathrm{PI}(3,4,5)-\mathrm{P} 3$ becomes $\mathrm{PI}(3,4,5)-\mathrm{P} 2$, leading to inactivation of AKT (13). The tumor suppressor proteins (PTEN and SHIP2), the proline-rich Akt substrate of $40 \mathrm{kDa}$ (PRAS40), and the tuberous sclerosis complex proteins (TSC1/2) are all negative regulators of mTOR (24). The phosphorylation of AKT can decrease this negative regulation, thus verifying that $\mathrm{AKT}$ aids in suppressing the negative regulatory factors of mTOR (25). However, more than one signaling pathway (other than PI3K/AKT) is responsible for the regulation of mTOR (26). Hunger and stress responses can result in the dephosphorylation of the autophagy-related protein Beclin-1, which inactivates mTOR and induces autophagy (26). AKT can promote the activation of mTOR as an upstream effector molecule of mTORC1. In the present study, miR-138 overexpression significantly suppressed the PI3K/AKT/mTOR signaling pathway in A2058 cells.

As an upstream gene of AKT, PDK1 regulates the activities of AKT and other downstream kinases of PI3K/ AKT, which enables PDK1 to regulate a series of biological responses in cells by the PI3K/AKT signaling pathway (27). PDK1 is a serine/threonine kinase of the AGC protein kinase family. As an important transduction molecule of the PI3K signaling pathway, the binding of PDK1 with PIP3 plays an important role in physiological processes, including the activation of kinases (such as AKT), the control of cell responses by regulating a large number of AGC protein kinase family members, and cell metabolism, growth, proliferation and survival (28). Research on PDK1 in recent years has demonstrated the following: firstly, as a type of regulatory kinase, PDK1 can activate 23 types of downstream kinases; secondly, PDK1 can continuously phosphorylate substrates due to its constant self-activation, which stimulates conformational changes in the substrate to expose anchor points and increase binding with PDK1, and this is an important mechanism by which PDK1 controls a large number of AGC kinase activities; thirdly, PDK1 is a type of single-copy sequence gene that is applicable to genetic analysis, and research progress has been made by use of the transgenic mouse model; and fourthly, inhibitors of PDK1 may be useful in the treatment of diseases such as diabetes and cancer, due to its key roles in numerous signal transduction pathways $(15,29,30)$. In the present study, the PI3K inhibitor LY294002 suppressed $\mathrm{PI} 3 \mathrm{~K} / \mathrm{AKT} / \mathrm{mTOR}$ signaling, inhibited proliferation, induced apoptosis and caspase-3 activity, promoted LC3 and Bax protein expression, and decreased PDK1 protein expression in A2058 cells following miR-138 overexpression. Collectively these data suggest that LY294002 may suppress the growth and metastasis of MM. Similarly, Liu et al (31) reported that miR-138 suppresses airway smooth muscle cell proliferation by targeting PDK1 in the PI3K/AKT signaling pathway.

In conclusion, we demonstrated the clinical significance of miR-138 in patients with MM through its targeting of PDK1 expression in the PI3K/AKT/mTOR autophagy signaling pathway. These results may provide insight into the mechanisms of miR-138 as a therapeutic target for the treatment of MM.

\section{References}

1. Greene JM, Schneble EJ, Jackson DO, Hale DF, Vreeland TJ, Flores M, Martin J, Herbert GS, Hardin MO, Yu X, et al: A phase I/IIa clinical trial in stage IV melanoma of an autologous tumor-dendritic cell fusion (dendritoma) vaccine with low dose interleukin-2. Cancer Immunol Immunother 65: 383-392, 2016.

2. Nagaoka T, Kiyohara Y, Koga H, Nakamura A, Saida T and Sota T: Modification of a melanoma discrimination index derived from hyperspectral data: A clinical trial conducted in 2 centers between March 2011 and December 2013. Skin Res Technol 21: 278-283, 2015.

3. Grob JJ, Amonkar MM, Karaszewska B, Schachter J, Dummer R, Mackiewicz A, Stroyakovskiy D, Drucis K, Grange F, ChiarionSileni V, et al: Comparison of dabrafenib and trametinib combination therapy with vemurafenib monotherapy on health-related quality of life in patients with unresectable or metastatic cutaneous $B R A F$ Val600-mutation-positive melanoma (COMBI-v): Results of a phase 3, open-label, randomised trial. Lancet Oncol 16: 1389-1398, 2015.

4. Mohr P, Hauschild A, Trefzer U, Enk A, Tilgen W, Loquai C, Gogas H, Haalck T, Koller J, Dummer R, et al: Intermittent high-dose intravenous interferon alfa- $2 b$ for adjuvant treatment of stage III melanoma: Final analysis of a randomized phase III dermatologic cooperative oncology group trial. J Clin Oncol 33: 4077-4084, 2015.

5. Varamo C, Occelli M, Vivenza D, Merlano M and Lo Nigro C: MicroRNAs role as potential biomarkers and key regulators in melanoma. Genes Chromosomes Cancer, 2016.

6. Li JY, Zheng LL, Wang TT and Hu M: Functional annotation of metastasis-associated microRNAs of melanoma: A meta-analysis of expression profiles. Chin Med J 129: 2484-2490, 2016.

7. Mannavola F, Tucci M, Felici C, Stucci S and Silvestris F: miRNAs in melanoma: A defined role in tumor progression and metastasis. Expert Rev Clin Immunol 12: 79-89, 2016.

8. Jayawardana K, Schramm SJ, Haydu L, Thompson JF, Scolyer RA, Mann GJ, Müller S and Yang JY: Determination of prognosis in metastatic melanoma through integration of clinico-pathologic, mutation, mRNA, microRNA, and protein information. Int J Cancer 136: 863-874, 2015.

9. Aftab MN, Dinger ME and Perera RJ: The role of microRNAs and long non-coding RNAs in the pathology, diagnosis, and management of melanoma. Arch Biochem Biophys 563: 60-70, 2014.

10. Meng XX, Yao M, Zhang XD, Xu HX and Dong Q: ER stressinduced autophagy in melanoma. Clin Exp Pharmacol Physiol 42: 811-816, 2015. 
11. Lima RT, Sousa D, Paiva AM, Palmeira A, Barbosa J, Pedro M, Pinto MM, Sousa E and Vasconcelos MH: Modulation of autophagy by a thioxanthone decreases the viability of melanoma cells. Molecules 21: pii: E1343, 2016.

12. Caporali S, Alvino E, Lacal PM, Levati L, Giurato G, Memoli D, Caprini E, Antonini Cappellini GC and D'Atri S: Targeting the $\mathrm{PI} 3 \mathrm{~K} / \mathrm{AKT} / \mathrm{mTOR}$ pathway overcomes the stimulating effect of dabrafenib on the invasive behavior of melanoma cells with acquired resistance to the BRAF inhibitor. Int $\mathrm{J}$ Oncol 49: 1164-1174, 2016.

13. Peng Y, Li L, Huang M, Duan C, Zhang L and Chen J: Angiogenin interacts with ribonuclease inhibitor regulating PI3K/AKT/ mTOR signaling pathway in bladder cancer cells. Cell Signal 26 : 2782-2792, 2014.

14. Guo Y, Chang H, Li J, Xu XY, Shen L, Yu ZB and Liu WC: Thymosin alpha 1 suppresses proliferation and induces apoptosis in breast cancer cells through PTEN-mediated inhibition of $\mathrm{PI} 3 \mathrm{~K} / \mathrm{Akt} / \mathrm{mTOR}$ signaling pathway. Apoptosis 20: 1109-1121, 2015.

15. Liu Y, Gao X, Deeb D and Gautam SC: Oleanane triterpenoid CDDO-Me inhibits Akt activity without affecting PDK1 kinase or PP2A phosphatase activity in cancer cells. Biochem Biophys Res Commun 417: 570-575, 2012.

16. Scortegagna M, Ruller C, Feng Y, Lazova R, Kluger H, Li JL, De SK, Rickert R, Pellecchia M, Bosenberg M, et al: Genetic inactivation or pharmacological inhibition of Pdk1 delays development and inhibits metastasis of Braf ${ }^{\mathrm{V} 600 \mathrm{E}}:: \mathrm{Pten}^{-/-}$melanoma Oncogene 33: 4330-4339, 2014.

17. Li W, Song R, Fang X, Wang L, Chen W, Tang P, Yu B, Sun Y and $\mathrm{Xu}$ Q: SBF-1, a synthetic steroidal glycoside, inhibits melanoma growth and metastasis through blocking interaction between PDK1 and AKT3. Biochem Pharmacol 84: 172-181, 2012.

18. Lee SJ, Kim TM, Kim YJ, Jang KT, Lee HJ, Lee SN, Ahn MS, Hwang IG, Lee S, Lee MH, et al: Phase II trial of nilotinib in patients with metastatic malignant melanoma harboring KIT gene aberration: A multicenter trial of Korean Cancer Study Group (UN10-06). Oncologist 20: 1312-1319, 2015.

19. Guo J, Si L, Kong Y, Flaherty KT, Xu X, Zhu Y, Corless CL, Li L, Li H, Sheng X, et al: Phase II, open-label, single-arm trial of imatinib mesylate in patients with metastatic melanoma harboring $c$-Kit mutation or amplification. J Clin Oncol 29: 2904-2909, 2011.

20. Wang R, Jing G, Lv J, Song H, Li C, Wang X, Xia W, Wu Y, Ren $G$ and Guo W: Interferon- $\alpha-2 b$ as an adjuvant therapy prolongs survival of patients with previously resected oral muscosal melanoma. Genet Mol Res 14: 11944-11954, 2015.

21. Shao H, Cai L, Moller M, Issac B, Zhang L, Owyong M, Moscowitz AE, Vazquez-Padron R, Radtke F and Liu ZJ: Notch1-WISP-1 axis determines the regulatory role of mesenchymal stem cell-derived stromal fibroblasts in melanoma metastasis. Oncotarget 7: 79262-79273, 2016.
22. Ndoye A and Weeraratna AT: Autophagy - An emerging target for melanoma therapy. F1000Res pii: F1000 Faculty Rev-1888, 2016.

23. Stojcheva N, Schechtmann G, Sass S, Roth P, Florea AM, Stefanski A, Stühler K, Wolter M, Müller NS, Theis FJ, et al: MicroRNA-138 promotes acquired alkylator resistance in glioblastoma by targeting the Bcl-2-interacting mediator BIM. Oncotarget 7: 12937-12950, 2016.

24. Kou Y, Li L, Li H, Tan Y, Li B, Wang K and Du B: Berberine suppressed epithelial mesenchymal transition through cross-talk regulation of PI3K/AKT and RAR $\alpha / R A R \beta$ in melanoma cells. Biochem Biophys Res Commun 479: 290-296, 2016.

25. Zhao G, Han X, Zheng S, Li Z, Sha Y, Ni J, Sun Z, Qiao S and Song Z: Curcumin induces autophagy, inhibits proliferation and invasion by downregulating AKT/mTOR signaling pathway in human melanoma cells. Oncol Rep 35: 1065-1074, 2016.

26. Wei BR, Michael HT, Halsey CH, Peer CJ, Adhikari A, Dwyer JE, Hoover SB, El Meskini R, Kozlov S, Weaver Ohler Z, et al: Synergistic targeted inhibition of MEK and dual $\mathrm{PI} 3 \mathrm{~K} / \mathrm{mTOR}$ diminishes viability and inhibits tumor growth of canine melanoma underscoring its utility as a preclinical model for human mucosal melanoma. Pigment Cell Melanoma Res 29: 643-655, 2016.

27. Cordón-Barris L, Pascual-Guiral S, Yang S, Giménez-Llort L, Lope-Piedrafita S, Niemeyer C, Claro E, Lizcano JM and Bayascas JR: Mutation of the 3-phosphoinositide-dependent protein kinase-1 (PDK1) substrate-docking site in the developing brain causes microcephaly with abnormal brain morphogenesis independently of Akt, leading to impaired cognition and disruptive behaviors. Mol Cell Biol pii: MCB.00230-16, 2016.

28. Guo JP, Coppola D and Cheng JQ: IKBKE protein activates Akt independent of phosphatidylinositol 3-kinase/PDK1/mTORC2 and the pleckstrin homology domain to sustain malignant transformation. J Biol Chem 291: 22853, 2016.

29. Jung Y, Yi YS, Yoo DS, Kim JH, Yang WS, Lee J, Park KW, Kweon DH, Hong S and Cho JY: 8-(Tosylamino)quinoline inhibits tumour progression through targeting phosphoinositide-3-kinase/ Akt pathway. Pharmazie 68: 146-152,2013.

30. Medina JR: Selective 3-phosphoinositide-dependent kinase 1 (PDK1) inhibitors: Dissecting the function and pharmacology of PDK1. J Med Chem 56: 2726-2737, 2013.

31. Liu Y, Yang K, Sun X, Fang P, Shi H, Xu J, Xie M and Li M: MiR-138 suppresses airway smooth muscle cell proliferation through the PI3K/AKT signaling pathway by targeting PDK1. Exp Lung Res 41: 363-369, 2015 